\title{
Effective robust optimal control system for a lamellar pasteurization-cooling unit under the conditions of intense external perturbations
}

\author{
Nataliia Lutska ${ }^{1}$, Nataliia Zaiets ${ }^{2}$, \\ Lidiia Vlasenko ${ }^{1}$, Volodymyr Shtepa ${ }^{3}$
}

1 - National University of Food Technologies, Kyiv, Ukraine

2 - National University of Live and Environment Sciences of Ukraine, Kyiv, Ukraine

3 - Polessky State University, Pinsk, Belarus

Keywords:

Robust

Optimal

2-riccati

Uncertainties

H2-regulator

Article history:

Received 04.06.2018

Received in revised form 19.08.2018

Accepted 28.09.2018

Corresponding author:

Nataliia Lutska

E-mail:

lutskanm2017@

gmail.com

DOI: $10.24263 / 2304-$

974X-2018-7-3-15

\section{Abstract}

Introduction. Investigated robust optimal control system of pasteurization-cooling unit, functioning under conditions of intensive external disturbances, information and structural uncertainty in order to improve the efficiency.

Materials and methods. Methods of system analysis, mathematical statistics and identification used to construct a mathematical model of the control object. For the synthesis of the robust optimal controller, the 2-Riccati approach $\mathrm{H}_{2}$ control problems is applied, and to reduce the order of the received regulator - method for balanced model reduction.

Results and discussion. The work of the pasteurization unit is considered, since it is a key component of dairy production. Since the results of the operation of traditional automated systems depend on the intensity and quality of information flows, microprocessor technology, algorithmic and software, it is proposed to supplement them with robust optimal controllers based on the 2-Riccati approach. Identified a mathematical model of the object, which consists of linear differential equations of the seventh order, it is possible to synthesize a robust optimal controller of the 6th order. Performance indicators are calculated by comparing the synthesized system and the system with local regulators. In a robust optimal system, the magnitude of uncertainty increased fourfold, and the dispersion of control signals decreased fourfold, which proves its energy efficiency.

Conclusion. The performed modeling and calculation of qualitative characteristics showed a significant advantage of the synthesized system in front of systems with local regulators. 


\section{Introduction}

During Industry 4.0, when industrial enterprises grow into Smart Factory, combine advanced production technologies, automated production, communications and information technologies, the issue of improving automated control systems of technological processes based on intelligent approaches is relevant $[1,25]$. However, in terms of automation, such managing facilities have common problems of mismatch in temperature and quality values, uneven consumption and temperature of the incoming flow, as well as other internal and external factors which negatively affect the quality of the output product and the energy efficiency of the whole system. All these factors can be described in the uncertainty range of the object, and then it is possible to construct a robust optimal control system under one correctly selected criterion [2-5].

For production objects with significant uncertainties of the use of $\mathrm{H}_{2}-$ or $\mathrm{H}_{\infty}$-norms of the Hardy space of the closed system transfer function as a control criterion are effective for the proper types of input and output signals, whereby the system obtains the property of low-sensitivity (robustness) towards internal and external object changes [6]. The control device thus synthesized has a high order, and as a result, the complexity of the software implementation.

Since the object of study works under conditions of parametric and structural uncertainty, as well as under the influence of intense external disturbances, the goal of the work is to increase the efficiency of its control system by synthesizing an effective control device based on robust optimal practices.

\section{Materials and methods}

According to the purpose of the article, the following approaches and methods were used: system approach, statistical analysis, parametric identification of dynamic mathematical models, synthesis of the $\mathrm{H}_{2}$-regulator in the 2-Riccati approach, reduction of mathematical models, mathematical modeling. Using the system approach [7, 8], the theoretical bases of the study, in particular, the orderly and structure of the model of the control object are well-documented. The statistical analysis of process variables substantiated the essence of uncertainties, and also helped shape the form of weight transfer functions for external perturbations [9, 10]. Identification methods [11, 12] are used to obtain approximate parameters of the mathematical model of an object with multiplicative uncertainty. The synthesized $\mathrm{H}_{2}$-regulator differs from the classical one in that additional input and output matrix transfer functions are used [13-15]. The latter are formed on the basis of a compromise between the quality of the system in nominal mode, the energy saving of the system and the robustness to internal changes in the object. The introduction of matrix weight transfer functions led to an increase in the order of the synthesized regulator, therefore a number of methods for reducing the order of the regulator were subsequently used [16-20].

Based on the results of mathematical modeling of systems with robust and local regulators $[21,22]$, the main characteristics of quality and stability were calculated and the response graphs of the systems were obtained for various types of perturbations. 


\section{Results and discussion}

\section{Analysis of the object of control, a mathematical model of an object}

Modern dairy plants throughout the country are mostly equipped with lamellar pasteurization-cooling units of various types and constructions. A lamellar pasteurizationcooling unit is intended for continuous heating and pasteurizing of milk, cream and other liquid food products without losing the main properties such as protein, sugar and molecules of fat. The concept of their performance lies in the series flow from one tank into another, gradually mixing with the already heated milk, and then the milk is heated and maintained during an appropriate period of time at the temperature of pasteurization. After heating, the product is cooled and forwarded to other stages of processing or packaging.

Figure 1 shows a simplified scheme of production flows of a typical lamellar pasteurization-cooling unit. Here $t_{1 \mathrm{r}}$ is the temperature of incoming raw milk, $t_{1 \mathrm{p}}$ is the temperature of pasteurized milk, $t_{1 \mathrm{w}}$ is the temperature of the heated milk, $t_{1 \mathrm{rec}}$ is the temperature of recuperated milk, $t_{10}$ is the temperature of cooled pasteurized milk, $t_{2 \mathrm{~h}}$ is the temperature of hot water, $t_{2 \mathrm{c}}$ is the temperature of cooled water, $m_{1}$ is the consumption of milk, $m_{2}$ is the consumption of hot water, $m_{3}$ is the consumption of iced water, $m_{4}$ is the consumption of steam.

Automatic temperature regime control of a lamellar pasteurization-cooling unit is reduced to two local loops of temperature control for pasteurized and cooled milk (PID or PI) with a stabilized input flow. In terms of automation, the control object is multiplyconnected, transient; the coordinates of the state are divided by spatial value, also, the object operates under permanent intensive external disturbances which are characterized by both deterministic and random components, the latter having a pigmented spectrum. In addition to the external perturbations which are shown in Figure 1, the quality of the output product is influenced by the acidity of the input product and the ambient temperature, which are not included into the control loop. Consequently, the use of the local regulators leads to quality degradation of the output product, and in some cases leads to repasteurization of the processed milk.

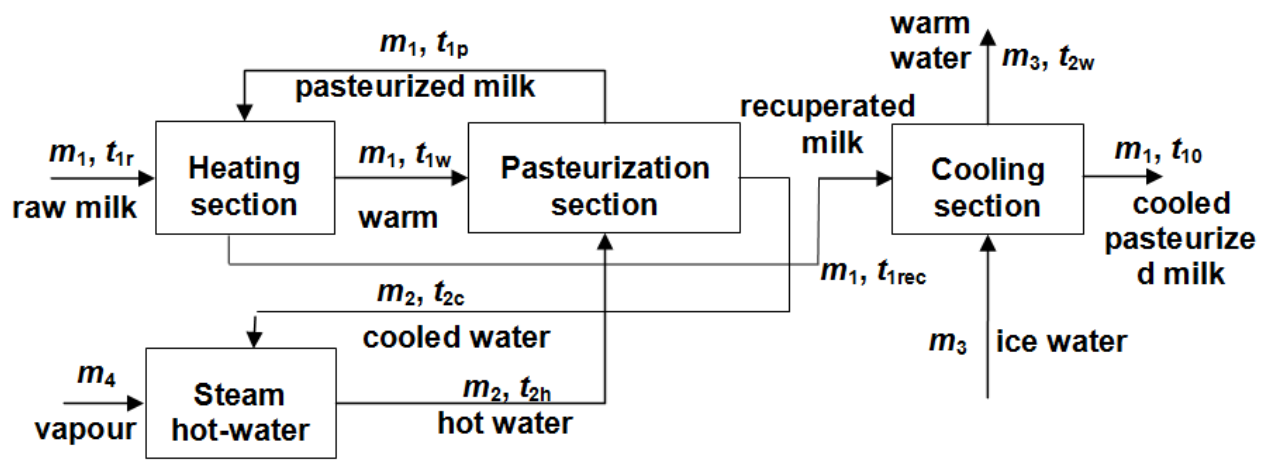

Figure 1. Scheme of the production flows in a lamellar pasteurization-cooling unit 
The problem of developing a mathematical model of a production process control object is still being controversial. For the synthesis of an effective control system, a mathematical model is to describe the object as best as possible, which leads to large orders of systems of differential equations, a complex description of parameters and traceability of a large number of external and internal variables in a production object, and consequently, to the complex and lengthy control algorithm, whereas simplifying the mathematical model reduces the quality of control [22]. Therefore, an alternative in developing the mathematical model of a production control object is using different types of uncertainties, which, on the one hand, simplifies the nominal model of an object, while taking into consideration the allowance in such description and changes in the object itself, on the other hand.

A lamellar pasteurization-cooling unit can be simply described by a system of differential equations of the $7^{\text {th }}$-order with a multiplicative type of uncertainty, including both parametric changes in an object and unmodulated dynamics, which appeared as a result of reducing the order of differential equations. Let's convert the model of a lamellar pasteurization-cooling unit to the space of state variables, taking into consideration the task signals in addition to the perturbations:

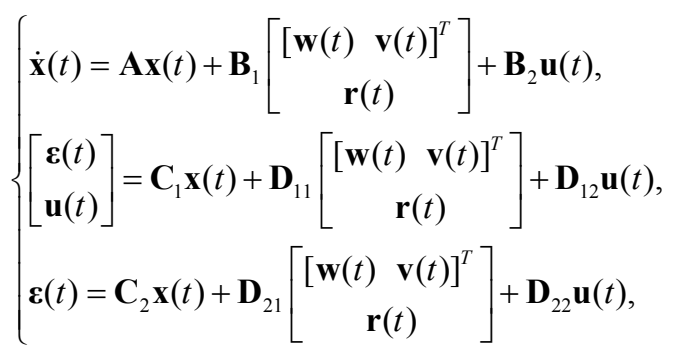

where $\quad \mathbf{x}(t)=\left[\Delta t_{\text {1rec }}, \Delta t_{1 w}, \Delta t_{1 p}, \Delta t_{2 c}, \Delta t_{10}, \Delta t_{2 w}, \Delta t_{2 h}\right]^{T}$ is a state coordinate vector; $\mathbf{u}(t)=\left[\Delta m_{3}, \Delta m_{4}\right]^{T}$ is a vector of control, $\mathbf{w}(t)=\left[m_{1}, m_{2}, \Delta t_{1 r}\right]$ is a vector of external perturbations; $\mathbf{v}(t)=\left[v_{1}(t), v_{2}(t)\right]$ is a vector of perturbations along the measuring circuit; $\mathbf{r}(t)=\left[\Delta r_{1}(t), \Delta r_{2}(t)\right]^{T}$ is a vector of the specified signals; $\mathbf{z}(t)=[\boldsymbol{\varepsilon}(t), \mathbf{u}(t)]^{T}$ is a vector of stabilized outputs; $\boldsymbol{\varepsilon}(t)=\mathbf{r}(t)-\mathbf{y}(t)$ is a vector of deviations from the specified signals; $\mathbf{y}(t)=\left[x_{3}(t), x_{5}(t)\right]^{T}$ is a vector of measurements.

All the matrix coefficients from (1) are determined on the basis of the aprior information about an object and as a result of identification [7-12]. The identification can be carried out by supplying pseudobinary signals to each input of the object and recording the output signals through the SCADA (Supervisory Control And Data Acquisition) program. The latter, through the PLC (Programmable Logic Controller) chain, is a field technology implemented by analog data transmission and industrial networks, receives information about the control object and executes a control signal. It is advisable to import the data into the Matlab software environment, where further processing will be carried out. Thus, for the A1-ОХЛ10 (OHL10) type lamellar pasteurization-cooling unit it is possible to determine and construct the basic matrix coefficients (1): 


$$
\begin{aligned}
& \mathbf{A}=10^{-2} \cdot\left[\begin{array}{ccccccc}
-23.31 & 0 & 20.75 & 0 & 0 & 0 & 0 \\
0 & -23.31 & 2.56 & 0 & 0 & 0 & 0 \\
0 & -2.25 & -22.47 & 0 & 0 & 0 & 24.72 \\
0 & 24.31 & 0 & -69.44 & 0 & 0 & 45.14 \\
3.07 & 0 & 0 & 0 & -10.57 & -54.02 & 0 \\
7.32 & 0 & 0 & 0 & 20.05 & -27.10 & 0 \\
0 & 0 & 0 & 19.16 & 0 & 0 & -19.16
\end{array}\right], \\
& \mathbf{B}_{1}=\left[\begin{array}{lll}
\mathbf{W}_{\mathbf{w}} \mathbf{B}_{1}^{\mathbf{w}} & \mathbf{B}_{1}^{\mathbf{v}} & \mathbf{B}_{1}^{\mathbf{r}}
\end{array}\right], \mathbf{B}_{1}^{\mathbf{v}}=\left[\mathbf{0}_{7 \times 2}\right], \mathbf{B}_{1}^{\mathbf{r}}=\left[\mathbf{0}_{7 \times 2}\right], \\
& B_{2}=\left[\begin{array}{cc}
0 & 0 \\
0 & 0 \\
0 & 0 \\
0 & 0 \\
0 & 0 \\
-4.078 & 0 \\
0 & 13.662
\end{array}\right] B_{2}=\left[\begin{array}{cc}
0 & 0 \\
0 & 0 \\
0 & 0 \\
0 & 0 \\
0 & 0 \\
-4.078 & 0 \\
0 & 13.662
\end{array}\right] \\
& \mathbf{C}_{1}=\left[\begin{array}{l}
-\mathbf{C}_{1}^{1} \\
\mathbf{0}_{2 \times 7}
\end{array}\right], \mathbf{C}_{1}^{1}=\left[\begin{array}{lllllll}
0 & 0 & 1 & 0 & 0 & 0 & 0 \\
0 & 0 & 0 & 0 & 1 & 0 & 0
\end{array}\right], \mathbf{C}_{2}=-\mathbf{C}_{1}^{1}, \\
& \mathbf{D}_{11}=\left[\begin{array}{ccc}
\mathbf{0}_{2 \times 3} & \mathbf{W}_{\mathbf{v}} \mathbf{I}_{2 \times 2} & \mathbf{I}_{2 \times 2} \\
& \mathbf{0}_{2 \times 7} &
\end{array}\right] ; \mathbf{D}_{12}=\left[\begin{array}{l}
\mathbf{0}_{2 \times 2} \\
\mathbf{I}_{3 \times 3}
\end{array}\right] ; \mathbf{D}_{21}=\left[\begin{array}{lll}
\mathbf{0}_{2 \times 3} & \mathbf{W}_{\mathbf{v}} \mathbf{I}_{2 \times 2} & \mathbf{I}_{2 \times 2}
\end{array}\right] ; \mathbf{D}_{22}=\left[\mathbf{0}_{2 \times 2}\right] .
\end{aligned}
$$

Here $\mathbf{0}_{i \times j}, \mathbf{I}_{i \times j}$ are zero and unity dimension matrices $i \times j$ respectively; $\mathbf{W}_{\mathbf{w}}, \mathbf{W}_{\mathbf{v}}$ are standardized weighting matrices of the intensity of external perturbations and noise measurements respectively. We might note that a control vector $\mathbf{u}(t)$ is additionally embedded into an output vector $\mathbf{z}(t)$ to restrict the signal of the former.

\section{Synthesis of a robust optimal regulator}

The functional chart of the control system is shown in Figure 2, where $\mathbf{G}(s), \mathbf{G}_{0}(s)$ are transfer functions of the uncertainty object and the nominal object accordingly, obtained from (1), and $\mathbf{K}(s)$ is a transferable function of the regulator, $\boldsymbol{\Delta}_{\mathbf{w}_{0}}(s)$ is a structural weighting uncertainty; $\mathrm{s}$ is a complex variable. Here, the mathematical model of the control object will be written as follows:

$$
\mathbf{G}(s)=\mathbf{G}_{0}(s)\left(\mathbf{I}+\boldsymbol{\Delta}_{\mathbf{W}_{0}}(s)\right), \quad\left\|\boldsymbol{\Delta}_{\mathbf{W}_{0}}(s)\right\|_{\infty}<\gamma,
$$

where $\gamma$ is the uncertainty range. 


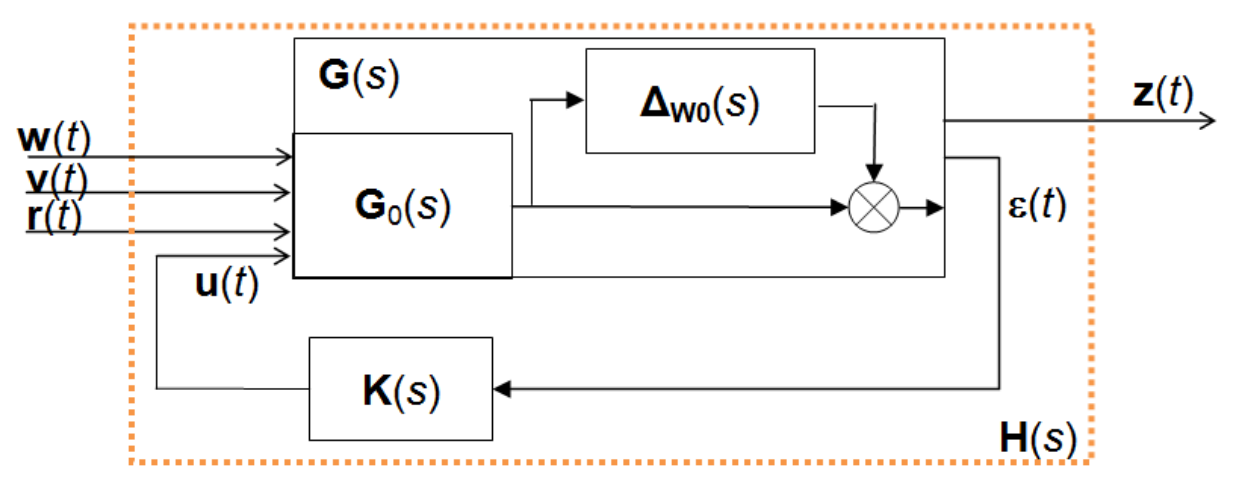

Figure 2. The functional chart of the control system

With regard to the above said, we can formulate the statement of the problem for the robust optimal control of a lamellar pasteurization-cooling unit: for a production object the mathematical model of which in the nominal mode is given by the system (1) with matrices (2), in the category of linear stabilizing controls

$$
\mathbf{u}=\mathbf{K}(s) \boldsymbol{\varepsilon}
$$

to find the optimal control which minimizes the specified quality criterion:

$$
J=\min _{\mathbf{K}(s) \in \Omega}\left\|\mathbf{W}_{L}(s) \mathbf{H}(s) \mathbf{W}_{R}(s)\right\|_{2},
$$

where $\mathbf{H}(s)$ is the transfer function of the closed system, $\Omega$ is the area of stabilizable controls; $\mathbf{W}_{L}(s), \mathbf{W}_{R}(s)$ are weighting matrices according to the outputs and inputs of the system. It is necessary to note that the weighting matrix $\mathbf{W}_{L}(s)$, specifies the quality of the system in the frequency range, and the matrix $\mathbf{W}_{R}(s)$ is a type of an input signal, in particular, one can specify a filter for an inhomogeneous signal spectrum. Thus, using criterion (5), the system minimizes the overall response pulse energy for the deterministic inputs, as well as the square root of the output dispersion for the specified dispersion of the input stochastic signal.

This problem has been solved in the state space for the standard $\mathrm{H}_{2}$ problem [13-15], based on the 2-Riccati approach, with the following weighting matrices:

$$
\begin{gathered}
\mathbf{W}_{L}=\operatorname{diag}\left(\frac{0.1}{50 s+1}, \frac{0.1}{125 s+1}, \frac{s}{500 s+1}, \frac{s}{1000 s+1}, 1,1\right) \\
\mathbf{W}_{R}=\operatorname{diag}\left(\frac{2.5}{2500 s+1}, \frac{1.5}{2000 s+1}, \frac{1}{200 s+1}, \frac{s}{s+0.1}, \frac{s}{s+0.1}, 1,1\right),
\end{gathered}
$$

where $\operatorname{diag}($ ) marks the diagonal matrix, while the linear regulator happens to be of the $11^{\text {th }}$ order. Analyzing the singular values of the transfer matrix of the regulator, it has been concluded that the regulator order can be reduced to the $6^{\text {th }}[16-20]$. The final regulator has 
been chosen and its order has been reduced by the method of balanced reduction according to the criterion:

$$
\left\|\mathbf{K}-\mathbf{K}_{r e d}\right\|_{\infty} \leq 2 \sum_{k+1}^{n} \sigma_{i},
$$

Where $\mathbf{K}(s), \mathbf{K}_{\text {red }}(s)$ are the transfer functions of the output and the reduced regulator respectively, with a marginal error of 0.0335 .

The regulator has been implemented in the range of state variables with the following matrices:

$$
\begin{aligned}
& \mathbf{A}^{K_{\text {red }}}=10^{-2} \cdot\left[\begin{array}{cccccc}
-0.88 & 0.52 & 0.33 & 0.59 & -5.65 & 0.67 \\
-3.51 & -388.41 & -265.61 & -35.26 & -8.09 & -18.69 \\
0.47 & 266.78 & -3.49 & 3.46 & 10.08 & -1.81 \\
0.25 & 52.73 & -6.25 & -1.14 & 28.34 & -2.42 \\
-5.42 & 13.64 & 1.05 & -11.09 & -184.56 & 130.86 \\
-0.39 & 30.69 & -1.60 & -0.24 & -125.39 & -3.48
\end{array}\right] \\
& \mathbf{B}^{K_{\text {red }}}=10^{-2} \cdot\left[\begin{array}{cc}
4.79 & 17.40 \\
149.45 & -4.68 \\
-12.23 & -2.08 \\
-6.10 & -1.54 \\
-0.17 & 60.97 \\
-4.84 & 5.18
\end{array}\right] \\
& \mathbf{C}^{K_{\text {rd }}}=10^{-2} \cdot\left[\begin{array}{cccccc}
18.04 & -2.10 & -4.11 & -6.27 & 60.96 & -6.80 \\
0.29 & 149.50 & 11.70 & 0.57 & 1.50 & 2.02
\end{array}\right], \mathbf{D}^{K_{\text {red }}}=\left[\mathbf{0}_{2 \times 2}\right] .
\end{aligned}
$$

\section{Results of the simulation}

The simulation of a system with a synthesized robust optimal regulator and local PID and PI regulators for a nominal model and a model with maximum multiplicative uncertainty has been conducted. The parameters of local regulators have been synthesized according to the criterion of maximum perturbation filtering. Signals of disturbances have been applied to the system input throughout the time frame, with external disturbances simulated by a stochastic and deterministic component, and at the time of $75 \mathrm{sec}$ the tasks from zero to $2{ }^{\circ} \mathrm{C}$ and $1{ }^{\circ} \mathrm{C}$ have changed respectively. The simulation results are shown in Fig. 3-5 and comparative characteristics are summarized in Table 1. 
Comparative characteristics of systems with $\mathrm{H}_{2}$ - and PID-PI regulators

\begin{tabular}{|c|c|c|}
\hline \multirow{2}{*}{ Characteristics } & \multicolumn{2}{|c|}{ Type of regulator } \\
\hline & $\mathrm{H}_{2}$-regulator & $P I D-P I$ regulators \\
\hline Maximum deviation & $\begin{array}{c}\varepsilon_{1}=2.1{ }^{0} \mathrm{C} \\
\varepsilon_{2}=1.0{ }^{0} \mathrm{C} \\
u_{1}=0.2 \mathrm{~kg} / \mathrm{sec} \\
u_{2}=0.9 \mathrm{~kg} / \mathrm{sec}\end{array}$ & $\begin{array}{c}\varepsilon_{1}=2.0{ }^{0} \mathrm{C} \\
\varepsilon_{2}=1.0{ }^{0} \mathrm{C} \\
u_{1}=1.0 \mathrm{~kg} / \mathrm{sec} \\
u_{2}=1.9 \mathrm{~kg} / \mathrm{sec}\end{array}$ \\
\hline Medium-quadratic deviation & $\begin{array}{c}D \varepsilon_{1}=0.2{ }^{0} \mathrm{C} \\
D \varepsilon_{2}=0.1{ }^{0} \mathrm{C} \\
D u_{1}=0.04 \mathrm{~kg} / \mathrm{sec} \\
D u_{2}=0.1 \mathrm{~kg} / \mathrm{sec}\end{array}$ & $\begin{array}{c}D \varepsilon_{1}=0.4{ }^{0} \mathrm{C} \\
D \varepsilon_{2}=0.1{ }^{0} \mathrm{C} \\
D u_{1}=0.2 \mathrm{~kg} / \mathrm{sec} \\
D u_{2}=0.4 \mathrm{~kg} / \mathrm{sec}\end{array}$ \\
\hline Stability margin & 0.08 & 0.12 \\
\hline$\left\|\mathbf{H}_{\mathbf{w \varepsilon}}(s)\right\|_{2}$ & 0.25 & 0.34 \\
\hline$\left\|\mathbf{H}_{\mathbf{v} \varepsilon}(s)\right\|_{2}$ & 1.02 & 2.25 \\
\hline$\left\|\mathbf{H}_{[\mathbf{w} \mathbf{v}] \varepsilon}(s)\right\|_{\infty}$ & 1.13 & 5.27 \\
\hline$\gamma$ & 0.56 & 0.13 \\
\hline$\|\mathbf{S}(s)\|_{\infty}$ & 1.63 & 5.29 \\
\hline
\end{tabular}

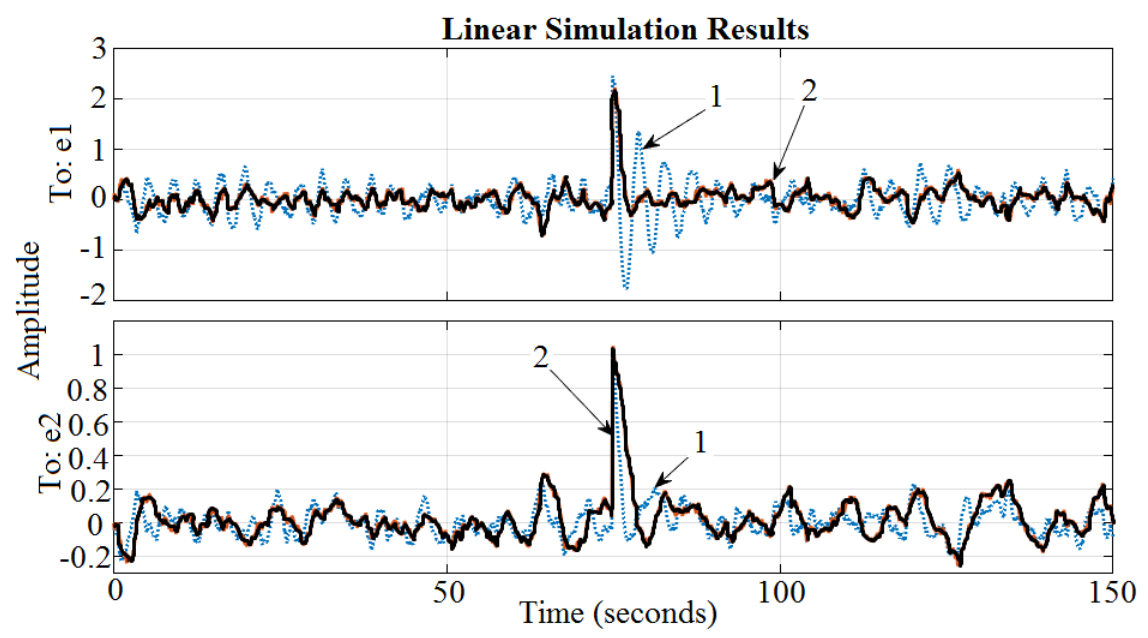

Figure 3. Deviation of pasteurization and cooling temperatures:

1 - System with PID-PI regulators; 2 -System with $\mathrm{H}_{2}$-regulators 


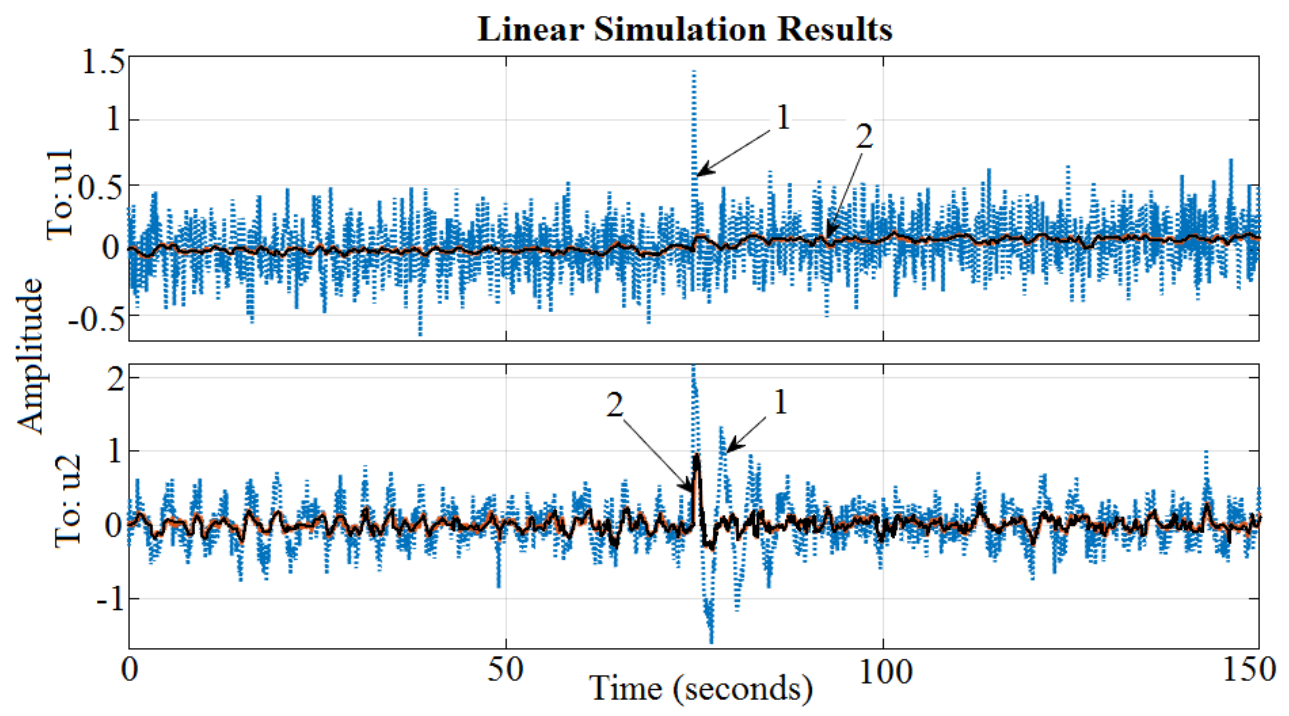

Figure 4. Deviation of control signals:

1 - System with PID-PI regulators; 2 - System with $\mathrm{H}_{2}$-regulators
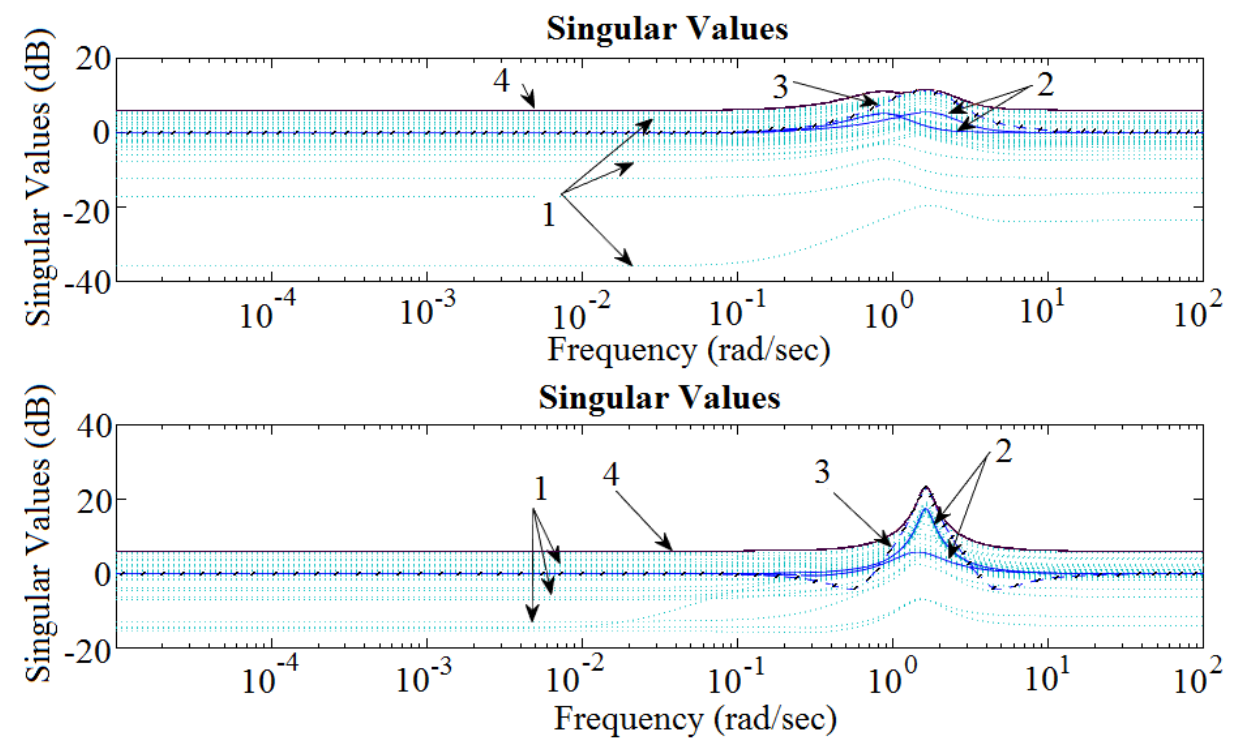

Figure 5. Singular values plot of dynamic system with uncertainty:

1 - System with sampled uncertainty; 2 - Nominal system; 3 - System with worst uncertainty; 4 - Worst-case gain (upper bound) 
The $\mathrm{H}_{2}$-regulator system shows better quality and energy-efficient performance, since the norms characterizing the quality of the system have decreased almost twice, and the scope of uncertainty has increased fourfold. In addition, the $\mathrm{H}_{\infty}$-norms of the sensitivity transfer functions $\mathbf{S}(s)$ for such a system are lower has tripled, which confirms the robust properties of the system. Also, the dispersion of the first regulated quantity was reduced twice, and the dispersion of control signals decreased respectively five and four times. In Fig. 5 is shown graphical worst-case gain analysis [21,22] synthesized system, in particular the curve of upper bound is the envelope produced by finding the highest gain within the uncertainty at each frequency. So, the time and frequency analysis of the system proved the effectiveness of the synthesized control system.

\section{Conclusion}

A lamellar pasteurization-cooling unit performs under the conditions of uncertainty of both the structure and parameters of the object, and intense external perturbations. Advance in efficiency of the control system of lamellar pasteurization-cooling units is possible by constructing a multidimensional optimal regulator in accordance with the $\mathrm{H}_{2}$-norm criterion. The mathematical model of the control object has been presented and reduced to the standard setting of the $\mathrm{H}_{2}$-optimization with the input and output weighting matrices while the synthesized regulator in the space of the state variables has been reduced to the $6^{\text {th }}$ order. The simulation of the system conducted by the $\mathrm{H}_{2}$-regulator has shown its effectiveness compared to the local PID-PI regulators.

Further research is aimed at automatically revealing the conditions for the applicability of robust-optimal regulators for building an information system to support management decisions.

\section{References}

1. Davis J., Edgar T., Porter J., Bernaden J. and M. Sarli (2012), Smart manufacturing, manufacturing intelligence and demand-dynamic performance, Computers \& Chemical Engineering, 47, pp. 145-156.

2. Bhattacharya S.P., Capellat H., Keel L.H. (2000), Robust Control: The Parametric Approach, Prentice Hall PTR.

3. Zhou, Kemin; Doyle C., John (1999), Essentials of Robust Control, Prentice Hall.

4. Morari Manfred; Zafiriou, Evanghelos (1989), Robust Process Control, Prentice Hall.

5. Ricardo S. Sánchez-Peñaand Mario Sznaier (1998), Robust Systems: Theory and Applications, Wiley, NewYork.

6. Ladanyuk A.P., Lutskaya N.N. (2016), Osobennosti zadach robastnogo upravleniia tekhnologicheskimi obieektami. Chast 1. Tekhnologicheskie obieekty i ikh matematicheskie modeli, Problemy upravleniia i informatiki, 5, pp. 16-23.

7. Pintelon R., Schoukens J. (2012), System Identification: A Frequency Domain Approach, Wiley, NewYork.

8. Skeppstedt A., Ljung L., Millnert M. (1992), Construction of composite models from observed data, Int. J. Control, 55(1), pp. 141-152.

9. Ninness B. M. (1993), Stochastic and Deterministic Modeling, University of Newcastle, NSW.

10. Milanese M. and Belforte G. (1982), Estimations theory and uncertainty intervals evaluation in the presence of unkown but bounded errors: Linear families of models and estimators, IEEE Trans. on Automatic Control, 27 pp. 408-414. 
11. Ljung L. (1999), System Identification: Theory for the User, Upper Saddle River.

12. Helmicki A.J. (1991), Control oriented system identification: A worst case / deterministic approach in $\mathrm{h}_{\infty}$, IEEE Transactions on Automatic Control, 36, pp. 11631176.

13. Doyle J.C., Glover K., Khargonekar P., Francis B. (1989), State-space solutions to standard $\mathrm{H}_{2}$ and $\mathrm{H}_{\infty}$ control problems, IEEE Trans. on Automat. Contr., 34 (8), pp. 831-847.

14. Khargonekar P. (1991), State-Space $H_{\infty}$ Control Theory and the LQG Problem, in Mathematical System Theory: the influence of R.E. Kalman, A.C. Antoulas, SpringerVerlag.

15. Glover K. (1984), All Optimal Hankel Norm Approximation of Linear Multivariable Systems, and Their L $\mu$-error Bounds, Int. J. Control, 39(6), pp. 1145-1193.

16. Safonov M.G., Laub A.J., Hartmann G. (1981), Feedback Properties of Multivariable Systems: The Role and Use of the Return Difference Matrix, IEEE Trans. of Automat. Contr., AC-26, pp. 47-65.

17. Safonov M.G., Chiang R.Y. (1989), A Schur Method for Balanced Model Reduction, IEEE Trans. on Automat. Contr., 34(7), pp. 729-733.

18. Safonov M.G., Chiang R.Y., Limebeer D.J.N. (1990), Optimal Hankel Model Reduction for Nonminimal Systems, IEEE Trans. on Automat. Contr., 35(4), pp. 496502 .

19. Zhou K. (1993), Frequency-weighted model reduction with $\mathrm{L}_{\infty}$ error bounds, Syst. Contr. Lett., 21, pp. 115-125.

20. Safonov M.G., Chiang R.Y. (1988), Model Reduction for Robust Control: A Schur Relative Error Method, International J. of Adaptive Control and Signal Processing, 2, pp. 259-272.

21. Blight J.D., Dailey R.L. and Gangsass D. (1994), Practical control law design for aircraft using multivariable techniques, International Journal of Control, 59(1), pp. 93-137.

22. Bates D. and Postlethwaite I. (2002), Robust Multivariable Control of Aerospace Systems, Delft University Press, Delft.

23. Korobiichuk I., Ladanyuk A., Vlasenko L., Zaiets N. (2018), Modern Development technologies and investigation of food production technological complex automated systems, International Conference on Mechatronics Systems and Control Engineering (ICMSCE), Amsterdam, Netherlands, pp. 52-57.

24. Iackymchuk T. (2013), Ukrainian Companies of Food Industry: Investment Activity and Factors Affecting the Results, Economics \& Sociology, 6(2), pp. 82-93, DOI: 10.14254/2071-789X.2013/6-2/8

25. Penchuk A. (2013), Dairy Industry in Ukraine: Evaluation of Business Efficiency, Economics and Sociology, 6(2), pp. 53-64, DOI: 10.14254/2071- 789X.2013/6-2 